\title{
Externalización y vinculaciones interempresariales desde la perspectiva gerencial $^{*}$
}

\author{
Lucena, Héctor** \\ Hernández Arias, Aymara***
}

\section{Resumen}

El objetivo general del presente artículo consiste en analizar las perspectivas gerenciales sobre la externalización y las vinculaciones interempresariales: sus razones, posibilidades y limitantes. Se trata de un estudio cualitativo de carácter exploratorio-descriptivo donde se trabaja bajo los supuestos del paradigma interpretativo que explican la realidad y el conocimiento de la misma a través de las interpretaciones y significados aportados por los actores involucrados en un fenómeno empresarial, incluyendo los investigadores. El método de investigación fue el estudio de caso y se utilizó el análisis de contenido temático para organizar y categorizar los datos fuentes (entrevistas focalizadas). El proceso de externalización y cooperativización emprendido surgió como un mecanismo de sobrevivencia y una estrategia de especialización para obtener flexibilidad organizativa y mayores niveles de productividad ante un entorno político-económico adverso y ante la rigidez de las regulaciones laborales. Una de las consecuencias fue la reestructuración de los procedimientos, normas y políticas internas de la empresa promotora para adaptarse a la dinámica de las vinculaciones entre la empresa y sus socios estratégicos.

Palabras clave: Externalización, cooperativas, vinculaciones interempresariales, perspectiva gerencial.

\section{Recibido: 12-06-07. Aceptado: 25-09-07}

Trabajo presentado en el V Congreso Latinoamericano de Sociología del Trabajo organizado por ALAST. Montevideo, Uruguay, 18 al 20 de abril de 2007. Versión revisada.

** Ingeniero en Informática. Magíster en Ingeniería Industrial. Estudiante del Programa de Doctorado en Estudios del Desarrollo (CENDES-UCV). Docente Asociado DAC-UCLA. E-mail: aymarah@yahoo.com.

*** Doctor en Ciencias Sociales de la Universidad de Glasgow. Coordinador del Doctorado en Ciencias Sociales de la Universidad de Carabobo. Valencia. Estado Carabobo. E-mail: hllucena@ hotmail.com - hlucena@postgrado.uc.edu.ve. 


\title{
Outsourcing and Inter-Company Links from Management's Perspective
}

\begin{abstract}
This article examines management perspectives regarding outsourcing and inter-company links: their reasons, possibilities and limiting factors. It is a qualitative study of an exploratorydescriptive character, carried out with assumptions from the interpretive paradigm which explains reality and knowledge of reality through interpretations and meanings contributed by the actors involved in an organizational phenomenon, including the researchers. The case study was used as the research method and thematic content analysis was utilized to organize and categorize the information sources (focused interviews). The process of outsourcing and co-operativization undertaken arose as a survival mechanism and a specialization strategy to obtain organizational flexibility and higher productivity levels in the face of an adverse political-economic environment and the inflexibility of labor regulations. One consequence was a restructuring of the internal procedures, norms and policies of the promoting company in order to adapt to the dynamics of the connections between the company and its strategic associates.
\end{abstract}

Key words: Outsourcing, cooperatives, intercompany links, management's perspective.

\section{Situación del contexto empresarial en la década de los ochenta: síntomas y causas}

La década de los ochenta comenzó con una profunda recesión global originando notables reducciones en el nivel de vida de Latinoamérica y, con el tiempo, cambios drásticos en las estrategias de desarrollo de la región representando un punto de quiebre que se manifestó en un período de crisis generalizada posterior a una etapa de crecimiento y bonanza. La convergencia de factores económicos y políticos desencadenó la crisis de la deuda del tercer mundo, dentro de lo que tuvo un peso crucial el alza abrupta de las tasas de interés por parte de los EEUU, todo lo que condujo a que esa década fuera definida por la CEPAL como "la década perdida". El año de 1982 marca el inicio de la crisis de la deuda externa para los países latinoamericanos aunado al agotamiento del modo de desarrollo económico y social basado en la sustitución de importaciones. Las políticas enmarcadas en lo que más tarde se conocería como el Consenso de Washington y los requisitos impuestos por los organismos de financiamiento internacionales implantaron una serie de reformas orientadas al mercado y una nueva estrategia, ahora conocida como neoliberalismo, la cual obligó a reestructurar la visión del proceso de desarrollo en sí. En el caso particular de Venezuela tuvo además un impacto muy importante, al principio de la década, la caída de los precios del petróleo (Clement y Pool, 1997; Mortimore y Peres, 2001; Gaviria, 2005).

En líneas generales, se pueden mencionar ciertos impactos negativos en el contexto de la denominada década perdida para Venezuela: en 1983 el país experimenta una crisis financiera y una 
devaluación significativa de la moneda. Para 1988 se agotan las reservas internacionales y se acumula una enorme deuda externa. EI PIB per cápita disminuye a todo lo largo de la década, mientras los ingresos reales, sueldos y salarios ajustados conforme a la inflación, disminuyen al nivel de los setenta. El desempleo tuvo un alza súbita y la inflación fue más alta en los ochenta que en las anteriores décadas a la par de una disminución drástica del crecimiento económico ${ }^{1}$.

En este contexto, las empresas agobiadas por un cambio en la política de competencia se han abocado a reducir costos, mejorar la eficiencia, reorganizar el uso de la mano de obra e implantar estrategias para lograr un mejor posicionamiento en el mercado. Aquellas que emprendieron cambios tuvieron resultados diversos: cada una con su historia de éxitos y fracasos según el camino seguido y el sector productivo al cual pertenecían. En el Cuadro 1 se muestran, según Marín (2004), las estrategias mayormente establecidas por las empresas según los diferentes tipos de ambiente. Cabe destacar que aunque no todas las empresas asumieron las mismas estrategias, la caracterización de cada escenario ofrece una panorámica de las más relevantes.

En este orden de ideas, la tendencia hacia una mayor flexibilidad es la solución que sobresale en el contexto de crisis actual (Bronstein, 1989; Urréa, 1999; Ozaki, 2000; Lucena, 2002; Hernández, 2005). Sin embargo, aunque se puede

\section{Cuadro 1}

\section{Estrategias competitivas en ambientes de globalización y competencia}

\section{Ambiente de Economía Cerrada. \\ Monopolio y protección \\ Ambiente de Competencia. \\ Enfoque Nacional}

\section{Estrategia competitiva en} defensa de la condición de monopolio y en la manipulación de precios. La revisión de la estructura y el mejoramiento de los procesos organizativos no forman parte de los programas estratégicos.
Estrategia competitiva basada en la obtención de los estándares en calidad y costos de la competencia nacional (igualdad de productos). Calidad centrada en atributos físicos del producto. Eficiencia centrada en procesos productivos.
Ambiente de Globalización y de competencia. Enfoque de Mercado Mundial

Estrategia competitiva basada en la superación de estándares de calidad y costos de la competencia (diferen-ciación del producto). La calidad centrada en atributos amplios asociados a la imagen corporativa. Eficiencia y calidad ampliada a los procesos organizacionales y a la revisión de estructuras empresariales.

Fuente: Elaboración propia con base en Marín (2004:22).

1 Para una exposición más detallada y presentación de indicadores que describen la situación anterior ver a CAN (1999), Sáez y Pineda (2004) y Arreaza y Pedauga (2006). 
concebir como una opción de adaptación y reestructuración para las empresas, al suponer costos sociales significativos y la necesidad de acordar e implantar nuevas regulaciones acordes con los nuevos esquemas, exige reflexiones sobre su impacto y sus beneficios para los actores sociales involucrados. Además, puede representar serias amenazas para los mismos, y para las cuales cuenten con los mecanismos (políticos, económicos y culturales) adecuados para afrontarlas. Los autores mencionados dan cuenta de esta situación.

\section{Sistematización del problema}

El punto de partida, a efectos del presente artículo, se ubica en la definición de externalización, refiriéndose "....al hecho de que la empresa saca fuera de su estructura cualquier actividad, sea de producción o de servicios" (Fundación Incyde et al., 2003:10), incluyendo empleados administrativos y trabajadores de planta, los cuales se organizan en empresas autónomas que establecen relaciones contractuales con la empresa origen (Pin y Suárez, 2004). El término se relaciona entonces con "...la transferencia de actividades que venía ejecutando el centro productivo terminal, a otros productores, especializados en los productos y/o servicios que se externalizan" (Lucena, 2004). Se hace una diferenciación con respecto a los términos de outsour- cing o subcontratación, los cuales contemplan "...aquellas partes específicas de la actividad productiva que son realizadas desde unidades productivas ajenas al que presenta finalmente el producto terminado en el mercado" (Fundación Incyde et al., 2003:10). En este último caso, las empresas vinculadas a través de la subcontratación o el outsourcing no poseen, por lo general, antecedentes de relaciones previas. De esta manera, el contratante hace la selección de las empresas especializadas sobre la base de criterios de experiencia, trayectoria, garantías de servicio, costos de los productos o servicios, imagen, entre otros. Dichos términos describen formas de flexibilización empresarial que según Iranzo y Richter (2004), son utilizadas en contextos donde el deterioro del mercado laboral (manifestado principalmente por la reducción de ingresos, desempleo y crecimiento del trabajo informal) induce a las empresas a buscar mecanismos alternativos para gestionar la fuerza de trabajo. Adicionalmente, implica para la empresa la cesión de obligaciones estipuladas en la Ley del Trabajo y por ende, la reducción de costos laborales ${ }^{2}$.

Por esta vía, la empresa se relaciona con otras organizaciones, las cuales sustituyen o proveen los productos o servicios que anteriormente se obtenian internamente, conformando de esta manera redes de empresas. Se establecen así vinculaciones interempresariales adicionales a las que mantenían tradicional-

Para más detalle sobre la flexibilización y su impacto en los aspectos laborales consultar Lucena $(2002,2007)$. 
mente con proveedores, clientes, gobierno e instituciones financieras ¿Qué se busca en este nuevo caso? Colaborar y cooperar. En fin, la empresa lo que requiere es obtener beneficio, aumentar la productividad y la eficiencia. La racionalidad tradicionalmente era económica. En el nuevo entorno intervienen motivaciones sociales (beneficios otorgados a los trabajadores, aspectos relacionados con el liderazgo, mejoramiento del proceso de trabajo en equipo, garantías de un ambiente adecuado y seguro de trabajo, entre otros). Otros aspectos relacionados con las vinculaciones interempresariales son la comunicación, el mejoramiento de los flujos de información y la creación de un ambiente propicio para las interacciones entre las partes.

El interés se orienta a la búsqueda de respuestas a interrogantes que establezcan ¿cuáles son las fórmulas de vinculación interempresarial que garantizan la sustentabilidad? Lo que se quiere es que la empresa se mantenga en el tiempo, no que la empresa tome una decisión que a la larga sea equivalente a firma de un contrato que conduzca al fracaso. Entonces, es necesario ubicar información teórica y empírica sobre los aspectos o factores que permitan mantener relaciones a largo plazo dentro del marco de los diversos procesos de reestructuración organizativa, entre ellos la externalización.

\section{Externalización, vinculaciones interempresariales y redes}

En el esquema brindado por la conformación de redes interempresariales, se ha producido un aumento de los procesos de externalización de actividades como vía para lograr estructuras organizacionales flexibles y más eficientes. En forma muy general, las partes relacionadas, por medio de contratos primordialmente comerciales, son: la empresa que externaliza y las nuevas organizaciones establecidas para proveer servicios o productos (IRCO et al., 2002). Estas últimas pueden estar integradas o no por los empleados que pertenecían a la empresa original.

Las razones para acometer estos procesos pueden ser estratégicas, tácticas, o simplemente una razón operativa. En relación a las razones estratégicas, permiten asumir un comportamiento competitivo a largo plazo y garantizar la flexibilización, agilización de las estructuras, creación de valor agregado, especialización, mejora en la calidad del servicio y aumento del nivel de aprendizaje organizacional mediante la adopción de prácticas empresariales distintas a las conocidas, búsqueda de ventajas políticas 0 acceso a tecnologías alternativas (Stuart, 2000; Canet et al., 2000; Ariño et al., 2001; IRCO et al., 2002). Las razones estratégicas se convierten en instrumentos para establecer relaciones cooperativas, interdependientes y a largo plazo para competir y sobrevivir en mercados globales. En cuanto a las razones operativas o tácticas, prevalecen la mejora de la eficiencia, reducción de costos de transacción, acceso a tecnología avanzada, búsqueda de economías de escala o el compartimiento de riesgos. Cada una de estas opciones representa oportunidades y amenazas para los acto- 
res involucrados que se hace indispensable analizar ${ }^{3}$.

Ariño et al. (2001) señalan ciertos elementos que definen la calidad de la alianza o sea atributos de la vinculación que inciden sobre su desempeño y éxito futuro, facilitan las transacciones e interacciones, motivan las actividades de valor agregado y ayudan a la resolución de conflictos. Dichos elementos se dividen en tres categorías: (a) Condiciones iniciales representadas por las características institucionales y demográficas, reputación, experiencia previa y procesos de negociación y acuerdo; (b) Interacciones entre los asociados materializadas por medio del cumplimiento de expectativas, comportamiento colaborativo, razones de cumplimiento $\mathrm{u}$ omisiones e intercambios de información y alertas anticipadas que aseguren la transparencia de las acciones; y, por último, (c) Eventos externos (sistémicos -cambios que afectan a la industria, sector o país de manera indiscriminada-, corporativos -actos ejecutados por alguna de las partes que están fuera del alcance de la alianza y que impactan los puntos de vista de los asociados-e individuales -actos realizados por representantes de las organizaciones asociadas que están fuera del alcance de la alianza y que afectan la manera como las partes ven la relación-). Un modelo para explicar el proceso de formación de una vinculación empresarial es referenciado por Keil (2000:19) y consiste en tres fases secuenciales: (a) Fase 1, de condiciones iniciales, cuyo propósito es reducir la incertidumbre a través de la fijación de expectativas y obligaciones; (b) Fase 2, establecimiento de la alianza a través del compromiso, enmarcado en las reglas, obligaciones, expectativas claras y la confianza reflejada en la reciprocidad de acciones; (c) Fase 3, de control e integración a nivel operacional y estratégico, contemplando además la dimensión social de la vinculación.

A lo largo de la evolución de una vinculación se pueden presentar conflictos provocados por la dinámica natural de los negocios o de las interacciones humanas que obligan a ubicar mecanismos para resolverlos fomentando un proceso de desarrollo y aprendizaje; también pueden presentarse confusiones en el cumplimiento de roles y funciones, los cuales es recomendable redimensionar o clarificar; debilidades en el sistema de indicadores de desempeño que indiquen desviaciones en los objetivos iniciales y en los procesos ejecutados en conjunto; además será necesario superar las conductas empresariales, grupales 0 individuales que provoquen reacciones negativas que puedan generar la interrupción 0

Monereo y Moreno (2004) categoriza la primera razón como "fisiológica", ya que representa una vía para lograr un adecuado nivel de adaptación, recurrir a recursos externos para mejorar las capacidades productivas y fomentar procesos de colaboración dentro de la red de empresas que se conforma. La segunda la clasifica como "patológica", donde los mayores impactos los reciben los trabajadores (utilización de la flexibilización numérica, contractual, productiva, etc.). 
desestabilización de la relación, entre ellas: conductas de dependencia o poder manifestadas por presión, imposición, amenaza o amonestación (Wilson y Vlosky, 1998). La dinámica de la vinculación dependerá también de las externalidades (tecnológicas, políticas y sociales), las cuales exigirán de las partes un reajuste continuo y la redefinición de las pautas de relación, a fin de aprovechar sus fortalezas y eliminar las debilidades, manteniendo así una configuración idónea para enfrentarlas.

Los factores en contra de las alianzas, que provocan fallas en su operacionalización, se pueden ubicar en aspectos relacionados con la rivalidad entre las firmas involucradas y con la complejidad de los procesos de gestión. Las alianzas pueden fallar por comportamientos oportunistas orientados a maximizar intereses individuales en detrimento de los intereses del colectivo por parte de alguno de los asociados, dificultades en la coordinación de acciones y en la alineación de las operaciones a largo plazo de las organizaciones implicadas (Ho y Ungson, 2001). Además resaltan inconvenientes $y$ desviaciones producidos por procesos de externalización, los cuales, si bien permiten flexibilizar la estructura organizativa mejorando la capacidad de respuesta al entorno y los niveles de productividad, pueden generar problemas relacionados con el empleo precario y fraude laboral (Lucena, 2004).

\section{Caso de estudio de empresa perteneciente al ramo de equipos, repuestos y servicios de maquinaria pesada en Venezuela (Período 1995-2004) ${ }^{4}$}

Con respecto al caso de estudio se hace referencia a una empresa ubicada en Venezuela que cuenta con sucursales en las principales ciudades del país: $\mathrm{Ca}$ racas, Maracaibo, Valencia, Barquisimeto, Puerto La Cruz y Puerto Ordaz; puntos de venta en Punto Fijo; operaciones on site en Guasare y La Guajira en el Edo. Zulia y en Ciudad Piar, Edo. Bolívar. Es una empresa con más de 75 años de funcionamiento en la distribución exclusiva de equipos y repuestos de maquinaria pesada provenientes de una empresa transnacional ubicada en los Estados Unidos. La misma ofrece repuestos originales, servicio especializado y entrenamiento como soporte a las inversiones de sus clientes. Posee tres divisiones de negocio: minería, energía y construcción. Como puede observarse la empresa esta vinculada a los principales sectores productivos, como proveedora de bienes y servicios indispensables para dichos sectores. De acuerdo a lo anterior, la empresa se puede ubicar en el sector transversal de la economía según la categorización presentada por Pirela (2005) vinculada directamente con grandes industrias de procesos (petrolera y petroquímica, ción titulada "La Externalización como habilitadora de redes interempresariales basadas en relaciones de colaboración”. CENDES-UCV. 2004. 
por ejemplo) por ser proveedora de bienes y servicios de dichos sectores.

\subsection{Antecedentes}

La empresa acometió, desde 1995, un proceso de reestructuración organizacional utilizando las figuras de empresas tradicionales jerárquicas y cooperativas como medio para externalizar actividades no medulares. Su forma organizacional era muy compleja lo que le daba poca flexibilidad para la toma de decisiones y hacia más lentos los procesos de negocios. La complejidad de la empresa, la cual se puede definir sobre la base del número de empleados, era alta, ya que antes de la reestructuración tenía aproximadamente 500 empleados dispersos a nivel nacional.

En un contexto generalizado de contracción de sus mercados principales y crisis la empresa poseía, entre otras, ciertas debilidades que la motivaron al cambio: un contrato colectivo muy pesado y una acción sindical debilitada ${ }^{5}$. Lo anterior se traducía en un obstáculo para la flexibilización debido al alto peso que la planta de trabajadores ejercía sobre la estructura de costos de la empresa. Según los entrevistados ${ }^{6}$ se presentaban inconvenientes, tales como: deterioro paulatino del capital y el patrimonio debido a que, independientemente del resultado del ejercicio económico, había que responder a un convenio colectivo, la empresa no tenía libertad de negociación, limitaciones excesivas establecidas por la ley del trabajo, fuga de talentos, requerimientos continuos de disminuir la carga de producción y por lo tanto, a despedir trabajadores, entre otros. Hay que advertir que la gerencia venezolana resiente el intervencionismo estatal y la legislación laboral tuteladora, ello se convierte en un fuerte incentivo a la búsqueda de opciones organizacionales elusivas.

\subsection{Diseño meta: intenciones y propósitos}

Se buscaba entonces la flexibilidad mediante la reducción de su estructura

Esta situación se presenta de manera generalizada en Venezuela. En palabras de Lucena (2004:188) "...con las transformaciones productivas ocurridas a partir de los procesos de reestructuración experimentados por los sistemas económicos, ha ocurrido una disminución de las tasas de sindicalización (...) se llegó a alcanzar tasas cercanas al $30 \%$ en la bonanza de los setenta (...) en los últimos diez años se mantiene en el 14\%"). Díaz (2000) por su parte, menciona algunas causas de la disminución de la sindicalización: la pérdida de eficiencia de la forma de actualización, marcada centralización de la toma de decisiones, debilitación estructural debido a orientaciones político-partidistas, a las que se puede adicionar, una representación gremial débil con tendencias a intereses individualistas caracterizadas por favorecer posiciones empresariales.

6 Las entrevistas fueron realizadas a 7 gerentes que formaron parte de los equipos de trabajo durante el inicio y desarrollo del proceso de externalización, en las áreas de staff, finanzas, legal, auditoría, recursos humanos sistemas e informática. Se seleccionaron de manera intencional porque tenían una amplia trayectoria en la empresa y poseían mayor conocimiento de las razones, objetivos, evolución e impactos iniciales del proceso. Se obviaron los nombres para mantener la confidencialidad de los entrevistados y de la empresa. 
organizativa. El diseño meta también estaba orientado en reducir el organigrama y concentrarse en una organización más plana que permitiera mayor rapidez y eficacia de todos los procesos de negocio. Adicionalmente, buscaba ganar la energía humana de los trabajadores, o sea, en lugar de ser un empresario exigiendo el cumplimiento de ciertas pautas y metas a los trabajadores, transformarse en un aliado de los mismos. Se pretendía propiciar un mayor compromiso y responsabilidad. El objetivo de reestructuración estaba fundamentado en la preocupación de los directivos en relación a la baja eficiencia del personal y la poca motivación a involucrarse en los resultados del negocio. Por ello surgieron ciertos requerimientos, entre ellos: cambiar la actitud hacia el trabajo, propiciar una mayor participación, crear un clima de trabajo de responsabilidad y compromiso para favorecer el cumplimiento de objetivos del negocio e infundir mayor confianza, sentido de pertenencia y de grupo, mejorar la coordinación y descentralizar la autoridad. El propósito era alinear las estructuras de incentivos. Otro aspecto orientador estaba representado por el logro de un mayor grado de especialización. Para la empresa promotora, especializarse cabalmente en la venta de equipos y repuestos, las cooperativas se enfocarían en el servicio al cliente y la atención post-venta y las empresas tradicionales a su área de acción profesional específica (asesoría especializada, actividades de mantenimiento, servicios empresariales, por ejemplo).

Es así como, en la fase inicial, el objetivo principal, desde la perspectiva interna de la empresa, era ubicar un tipo de relación entre empresarios, accionis- tas, empleados y trabajadores basada en otra lógica. Se pueden diferenciar dos etapas en esta fase: 1ra etapa de externalización a través del esquema de empresa tradicional jerárquica, la cual se ejecuta desde 1995. Actualmente figura como un primer intento de transformación organizacional. Se establecieron contratos con empresas formadas con ex-empleados que pertenecian a las Direcciones de Informática, Staff, Ventas, Minería y motores, Finanzas y Logística y postventa, así como también, personal de las Gerencias Nacionales, especialistas y analistas de mercadeo, ventas, y servicio. Estos contratos de servicio de naturaleza mercantil permitieron a la empresa delegar parte de las funciones en socios especializados. Se conformaron dos empresas dedicadas a la prestación de servicios informáticos y dos cuyo objetivo era ofrecer asesoría gerencial y técnica. Adicionalmente, se contrataron bajo la modalidad de outsourcing los servicios de empresas externas dedicadas a la asesoría empresarial en recursos humanos, servicios de call center con una empresa de telecomunicaciones y una de limpieza de instalaciones industriales. 2da etapa de externalización mediante cooperativas. La propuesta consistió en impulsar una alianza con una red de cooperativas y de esta forma garantizar la estabilidad en el entorno empresarial, así como también la estabilidad a nivel financiero y laboral. La gestación de dicho proyecto se inicia en 1997. De esta manera se realiza una propuesta a los empleados para que conformen cooperativas de manera que todas las sucursales y áreas de trabajo fuesen operadas por trabajadores asociados. Los miembros de las coopera- 
tivas se consideran socios estratégicos de la empresa y éstas se rigen por un contrato de servicio. En conjunto todos comparten los gastos, los riesgos del negocio y los beneficios sobre los resultados. La empresa mantiene la licencia de los productos y de las sucursales en cuanto a la infraestructura, máquinas y activos encargándose del pago de patentes e impuestos municipales.

Los cargos eliminados fueron los pertenecientes a la estructura de las sucursales y los relacionados con procesos externalizados. Para finales del 2004, existían 15 empleados en la estructura central de la empresa, las empresas asociadas agrupaban 160 empleados y las cooperativas 350. De esta manera, el $90 \%$ de empleados pertenecientes a las distintas sucursales del país pasaron a conformar asociaciones cooperativas con responsabilidad sobre los ingresos, sistemas de remuneración, procesos de aseguramiento de la calidad y gestión participativa de los negocios obtenidos en sus áreas geográficas de acción. El resto prefirió finalizar la relación de trabajo.

Se puede considerar en este punto una segunda fase, donde se establecen las condiciones para la alianza. Entre los aspectos que favorecieron la consolidación de esta fase se pueden mencionar: a) el apoyo firme, accesabilidad y compromiso constante del tren gerencial de la empresa; b) la contratación de un asesor experto que estableció un programa de inducción previo a la implantación del esquema; c) Reuniones de trabajo a nivel ejecutivo para evaluar las alternativas, decidir la configuración más idónea, diseñar e implantar el plan de reestructuración. Todos estos aspectos aseguraron que el proceso de llevara a cabo adecuadamente y con el mínimo de improvisaciones. También influyo el sentido de pertenencia y un alto nivel de confianza entre las partes gracias a una trayectoria de trabajo en conjunto de más de 8 años.

En el caso de las empresas se realizaron reuniones y sesiones de trabajo para definir los términos de las nuevas relaciones en cuanto al costo y alcance de los servicios. En las cooperativas, por su lado, se ejecuto un proceso de formación en cooperativas con una duración de 9 meses. Posteriormente, en sesiones de trabajo conjunto, en las cuales intervinieron futuros socios y asesores externos expertos en cooperativas, se definieron el costo del factor humano y los beneficios, tanto sociales como económicos de las partes involucradas ${ }^{7}$.

Los contratos de las cooperativas, de carácter mercantil, regulan principal-

Rubros del factor humano: Sumatoria de los ingresos individuales de los miembros = ingreso base $+\%$ ventas $+\%$ logro de objetivos (rendimiento por grupo de trabajo) más los apartados para: fondo de ahorro, ley de política habitacional, seguro social obligatorio, seguro de paro forzoso, fondo de inversiones, hospitalización, cirugía y maternidad, bono de actualización profesional, primas por hijo, útiles escolares, fiesta de fin de año, bono navideño, días de descanso, equivalente a prestaciones sociales, utilidades, vacaciones, apartado por retiro de la cooperativa, servicios funerarios, bono de alimentación, bono de productividad y desempeño. Incluye gastos de administración y contabilidad. 
mente las relaciones con la empresa promotora. Dichos contratos contemplan las políticas para establecer canales regulares de comunicación, los objetivos a perseguir, las instancias de diálogo y negociación, los procedimientos para la discusión de nuevos negocios, modificaciones de contratos de servicios y cambios en las políticas, los mecanismos para la notificación formal de denuncias, reclamos, investigación de hechos irregulares y arbitraje en casos de agotarse las instancias ordinarias.

En ambos casos los contratos no poseen cláusulas de exclusividad del servicio. Esto abre la posibilidad a los socios estratégicos de establecer negociaciones con otros clientes y expandir sus servicios y mercados metas.

La cooperativa se constituye en operadora de las instalaciones y los activos, ejecutando el trabajo que desempeñaban anteriormente, pero con una nueva figura que les garantiza el crecimiento profesional y económico. Así, la empresa reconoce el trabajo organizado como un aporte a la producción con el mismo status que el capital. En este punto es importante recalcar que las cooperativas operan en base a un espacio geográfico definido. De tal manera, no se establecen conflictos por comportamientos competitivos al momento de negociar contratos y proyectos entre las diversas cooperativas que conforman la red empresarial. Cada zona y región ofrece posibilidades de ne- gocio potenciales que son aprovechadas al máximo por cada cooperativa en las diversas áreas de acción del grupo. Si algún proyecto o negocio requiere de las habilidades y experiencia de miembros de cooperativas externas se establecen contratos temporales de servicio entre las mismas.

Uno de los incentivos para la vinculación fue la indexación de los salarios del personal de las empresas privadas y los beneficios obtenidos por las cooperativas en función de los niveles de venta netos obtenidos en conjunto. Lo anterior implicó aumentos hasta de un $200 \%$ en los ingresos. Se establecieron paquetes básicos incrementados en función del desempeño general de las organizaciones. Esto trajo como consecuencia que todos los miembros se sintieran involucrados en los procesos de negocio y entendieran que el cabal cumplimiento de sus funciones tendría un impacto en el desempeño general de la empresa aunque dicha función estuviera ubicada en el back-office (procesos administrativos de apoyo) o en el front-office (procesos relacionados con las ventas y el servicio al cliente).

Se pueden señalar los beneficios: (a) Desde la perspectiva de los socios cooperativistas. Los socios participan de las utilidades y beneficios por las ventas y la prestación de servicios. Los mismos administran de manera independiente los ingresos y gastos incurridos en la realización del trabajo ${ }^{8}$, convirtiéndose en ga- 
rantes de la eficiencia y la productividad general, ya que sus intereses se alinean con los de la empresa. Se fomenta un sentido de pertenencia y compromiso (b) Desde la perspectiva de la empresa promotora. Se logra mayor flexibilidad a través de la conformación de una red de empresas pequeñas bien articulada y se fomenta el sentido de comunidad de trabajo, de identidad y pertenencia entre las cooperativas y la empresa. (c) Desde la perspectiva de los clientes. Los mismos cuentan con un mejor servicio por el alto grado de motivación y participación de todo el personal y se cuenta con una empresa más sólida y adaptable al comportamiento volátil de la economía nacional.

\subsection{Discusión de resultados}

Uno de los resultados más relevantes es que la empresa pasó de tener poca flexibilidad y alta jerarquización vertical (concentrando la toma de decisiones y el control en pocas personas, lo cual propiciaba un ambiente laboral de alta dependencia, baja iniciativa y participación) a una empresa con una nueva filosofía gerencial, un tamaño optimizado y reducido y con una articulación coherente de sus relaciones internas y externas.
El trabajo organizado en torno a una red de empresas, con nuevas condiciones de trabajo y donde la responsabilidad es compartida por todos, ha permitido sortear los graves problemas económicos y políticos que enfrenta el país. Cada socio estratégico conoce el medio y está orientado a la búsqueda de nuevas oportunidades de negocio en función de la actividad productiva de su región y donde las condiciones de trabajo e ingreso sean optimas según criterios de aceptación logrados en consenso por integrantes de la asociación. Se establece de esta manera una alianza estratégica de socios de negocio orientada al éxito bajo una relación de ganar-ganar. Se puede considerar que se trató de una reestructuración concertada en donde los actores negociaron en el marco de una relación madura y de reconocimiento mutuo (Lucena, 2004).

A manera de resumen se presentan en el Cuadro 2 los efectos que la reestructuración acometida ha generado en los actores en opinión de los entrevistados.

En cuanto a los efectos generados, tanto a nivel de empresa como de cooperativas, ver el Cuadro 3.

mento substancial de los ingresos mensuales y anticipos porexcedentes, autonomía en la toma de decisiones, flexibilidad ante la dinámica del entorno empresarial, facilidad para adquirir formación y experiencia cultural, posibilidad de transformarse en empresarios, acuerdos en consenso, participación directa en el logro de resultados y en la mejora de los procesos y/o procedimientos operativos y administrativos, preparación en el área de manejo de cooperativas a través de diplomados ofrecidos por la Dirección de Recursos Humanos de la empresa promotora, por nombrar algunos. 


\section{Cuadro 2}

\section{Efectos sobre los actores involucrados en el caso bajo estudio}

Ejecutivos y empleados

- Los empleados del outsourcing pierden la estabilidad que antes tenían, debido a que los contratos dependen del grado de desempeño que posean y en algunos casos tienen carácter temporal según las necesidades de la empresa. Esto obliga a las empresas por outsourcing a ubicar otros clientes y nichos de actuación para ampliar su alcance.

- Se les exige fortalecer la capacidad de oír y respetar las opiniones de los socios estratégicos, sin imponer las propias o mantener actitudes de poder basadas en la autoridad por el cargo. Esto significa ajustarse a otro tipo de relación, empresa-socios estratégicos, en la cual la jerarquía no existe.

- Es imprescindible que diseñen sistemas de evaluación que ofrezcan indicadores de desempeño, los cuales evidencien el cabal cumplimiento de sus funciones y justifiquen su permanencia en la empresa y los ajustes necesarios en los niveles de salarios o costos de los contratos.

- Pérdida de poder de los ejecutivos. Esto ha causado problemas de adaptación en los mismos evidenciados por la renuncia de algunos por no entender el nuevo esquema de trabajo.

- Los socios estratégicos, representados por los cooperativistas, exigen y participan en las decisiones estratégicas del negocio. Lo anterior implica crear y fortalecer los espacios de participación y compartir la visión del negocio.

- Necesidad de delegar funciones y compartir responsabilidades.
- Poseen mayores responsabilidades por los deberes y obligaciones adquiridos. En ellos recae la responsabilidad de la administración de los bienes en comodato de la sucursal y la gestión de las operaciones diarias y calidad de los servicios que se prestan.

- Si no cumplen lo estipulado en los contratos, principalmente los parámetros de calidad, pueden causar la disolución del contrato de manera unilateral.

- En el nuevo esquema tienen autonomía y libertad de decidir cómo operan, definir prioridades, ubicar oportunidades de negocio, siempre y cuando cumplan con los lineamientos estipulados por la casa matriz en cuanto a calidad de servicio. Esta libertad de decisión también acarrea mayores responsabilidades y riesgos.

- Requieren fortalecer la visión de negocio.

- Se hace necesario que se fortalezcan las capacidades emprendedoras.

- Ya no son considerados individuos aislados sino un colectivo con mayores fortalezas.

Fuente: Elaboración propia. 


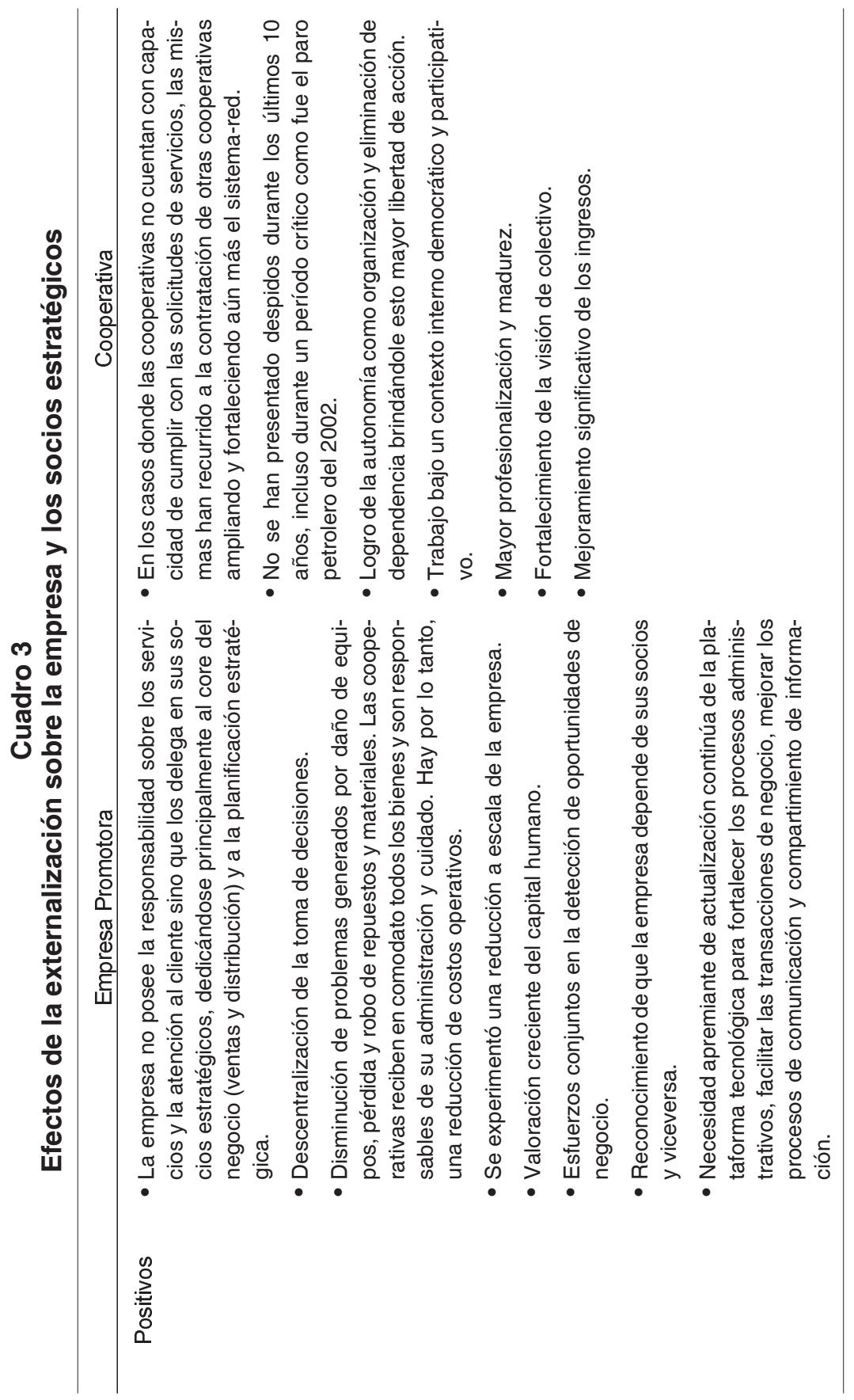




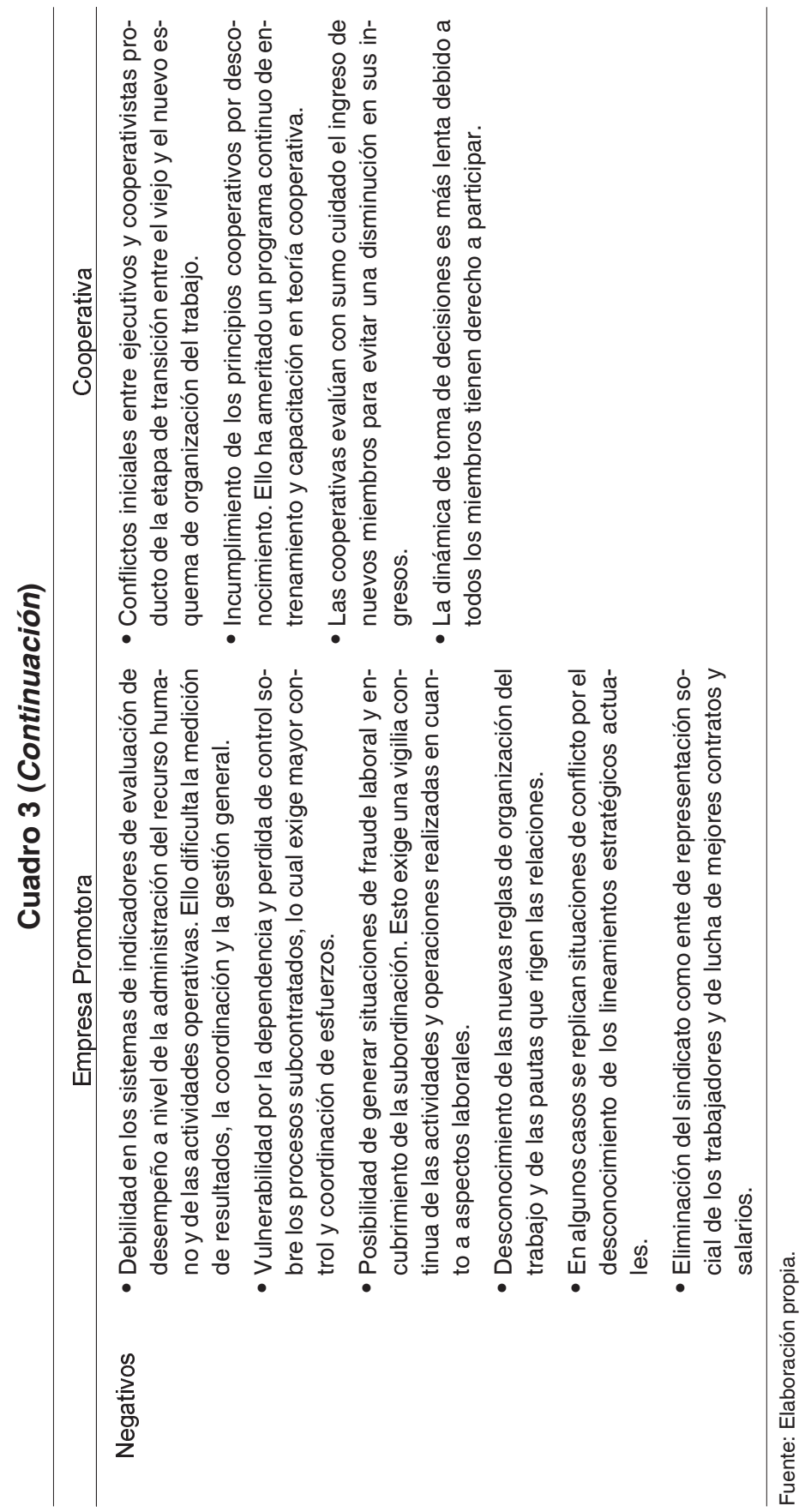




\section{Conclusiones y Recomendaciones}

En Venezuela y en diversos países existen posiciones contradictorias en cuanto al uso de la figura cooperativa como mecanismo de flexibilización por parte de las empresas. Se mencionan sobre todo los riesgos laborales representados en la derivación hacia figuras de subordinación y dependencia encubierta y que las organizaciones que conformen la red no sean realmente autónomas en sus funciones y decisiones. No todas las experiencias empresariales que han utilizado este esquema han sido exitosas. Entre los factores críticos de éxito del caso de estudio descrito se encuentran: respaldo irrestricto, reconocimiento del valor y potencial de las cooperativas por todos los miembros del sistema red, nivel profesional de los involucrados, reconocimiento de la importancia del concepto autonomía, confianza existente entre los miembros gracias a la amplia trayectoria de trabajo en conjunto, alta valoración del capital humano y monitoreo continuo de las vinculaciones interempresariales que permite detectar oportunamente desviaciones o efectos no deseados haciendo los correctivos necesarios.

El proceso de externalización y cooperativización emprendido surgió como un mecanismo de sobrevivencia y una estrategia de especialización para obtener flexibilidad organizativa y mayores niveles de productividad ante un entorno político-económico adverso y ante la rigidez de las regulaciones laborales. El mismo ha traído como consecuencia una reestructuración compleja de los procedimientos, normas y políticas internas de la empresa promotora para adaptarse a la dinámica de las vinculaciones entre la empresa y sus socios estratégicos. La decisión ha generado los resultados esperados y existe consenso, desde la perspectiva gerencial, que era el camino más adecuado. La prueba ácida la experimentaron en conjunto empresa-cooperativas, durante el paro petrolero de Diciembre 2002-Enero 2003, el cual afectó la mayoría de las actividades económicas y productivas del país. En otras circunstancias, con dos meses de paralización y aún encontrándose en un período de baja producción por razones estacionales, la empresa se hubiese visto forzada a despedir al menos $50 \%$ de los trabajadores, sin embargo, los miembros de las cooperativas reajustaron sus ingresos a baja en aproximadamente un $40 \%$ y lograron mantenerse hasta la reactivación con las reservas económicas que poseían. En el anterior esquema no hubiese sido posible.

Dicho proceso genero un período de transición, incertidumbre y cierta confusión mientras los miembros de la empresa promotora y de las organizaciones externalizadas asumían el nuevo esquema de trabajo. Por el lado de los ejecutivos, implico adaptarse a una dinámica de relaciones donde los criterios del poder por el cargo y por autoridad se disolvieron dándole paso a criterios basados en la participación, negociación, resolución de conflictos en consenso, donde el respeto y la tolerancia son valores necesarios. Aprender a compartir la visión del negocio con sus socios estratégicos implica fortalecer espacios para la discusión productiva y el trabajo grupal sin distinciones en cuanto a grado de responsabilidad y 
nivel profesional: todos tienen derecho a participar. Por el lado de los nuevos cooperativistas, significo el rompimiento de una relación de subordinación y dependencia hacia la reorganización radical del ambiente de trabajo.

Los entrevistados concuerdan en que los miembros de las organizaciones externalizadas cuentan con mejores condiciones laborales y sociales, pero también adquirieron mayores responsabilidades, haciendo necesario alcanzar madurez como profesionales y como personas integrantes de un colectivo. Lo anterior justifica la prioridad que le da la empresa a los programas de desarrollo profesional y capacitación en las áreas de gerencia, mejora de procesos, aspectos técnicos y educación cooperativa.

El período de transición se manifestó con cierta lentitud y contradicciones principalmente a raíz de problemas humanos, aunque no se puede hablar todavía de una consolidación del proceso, ya que la empresa ha requerido reestructuraciones continuas posteriores a la implantación del mismo y hasta la fecha la reorganización interna consume tiempo y recursos. Existen características culturales en las empresas que pueden obstaculizar incluso inhibir esfuerzos de reestructuración antes de ser implementados. Es responsabilidad de los promotores de la reestructuración empresarial y los participantes de las empresas externas, con las cuales posiblemente se establecerán relaciones estratégicas, tomar en cuenta las barreras potenciales y definir los mecanismos para eliminarlas.
Sin embargo, los actores han comprendido que las relaciones de las organizaciones que conforman el sistema-red son interdependientes y co-dependientes lo cual permite ponderar las decisiones $y$ acciones a realizar, ya que este tipo de esquema de trabajo, aunque es recomendable, sobre la base de las tendencias actuales en el entorno empresarial, también es altamente vulnerable. Se recomienda entonces que este tipo de estrategias empresariales, que tienen un alto impacto en la organización y en el componente humano, que requieren tiempo y recursos tecnológicos y financieros para su implantación, se enmarquen dentro de una visión integral del negocio, ya que de lo contrario caen en enfoques operativos orientados a la simple reducción de costos, lo cual pone en riesgo la sustentabilidad de las empresas.

En este orden de ideas, es importante entonces, orientar y propiciar investigaciones sobre aspectos empresariales hacia líneas de conocimiento que exploren a profundidad los nuevos elementos, relaciones y actores que están influyendo en la modificación radical de los procesos de negocio, aplicaciones empresariales y estructuras organizativas a partir del estudio de experiencias concretas que den cuenta de los aspectos culturales, políti$\cos$ y sociales particulares a cada realidad para crear modelos de alto desempeño capaces de competir y mantenerse en los cambiantes escenarios de la economía actual. 


\section{Referencias Bibliográficas}

Arreaza, Adriana y Pedauga (2006). Determinantes de los cambios en la productividad total de los factores en Venezuela. BCV. Serie Documentos de Trabajo. $N^{\circ} 71$. Fuente: www.bcv. org.ve (Consultado el 23-03-2007)

Ariño, Africa; J. de la torre y Smith, P. (2001). Relational quality: managing trust in corporate alliances. Draft. IESE España-The Anderson School at UCLA-Loyola Marymount University. USA.

Bronstein, Arturo (1989). La flexibilidad del trabajo. Panorama general. Ginebra. En UCLA. 1991. La flexibilización del trabajo. Un estudio internacional. pp. 13-51. Venezuela.

CAN (1999) . La evolución de las economías andinas y su competitividad. Documentos Estadísticos. Fuente: www.comunidadandina.org. (Consultado el 15-03-2007)

Canet,María, Peris, F y Redondo, A. (2000). El proceso estratégico asociado a la decisión de desintegrar actividades. $\mathbf{X}$ Congreso ACEDE. España. Fuente: www.acede.f2i.org (Consultado el 23-01-2004)

Clement, Norris y Pool, J. (1997). Economía. Enfoque América Latina. 4ta. Edic. McGraw Hill. México.

Díaz, Rolando (2000) . Sindicatos y nuevo escenario político en Venezuela. Nueva Sociedad. No. 169. pp. 153-161. Argentina. Fuente: www.nuso.org. (Consultado el 25-03-2007)

Fundación Incyde (2003). Consejo Superior de Cámaras de Comercio de España y Fondo Social Europeo. La subcontratación industrial en España y sus repercusiones en el empleo y en la creación de empresas. España. Fuente: www.subcont.com. (Consultado el 16-06-04).
Gaviria, Mario (2005). Neoliberalismo, "Consenso de Washington" y Reformas Estructurales en América Latina. Revista Contribuciones a la Economía. España. Fuente: http://www.eumed.net (Consultado el 05-04-07).

Hernández Arias, Aymara (2005). Flexibilización y organización del trabajo. Revista de Ciencias Sociales. Vol. XI. No. 2. pp. 256-276. Venezuela.

Ho, Seung and Ungson, G. (2001). Interfirm rivalry and managerial complexity: a conceptual framework of alliance failure. Organization Science. Vol. 12. No. 1.

Iranzo, Consuelo y Richter , J. (2004). La subcontratación laboral. Bomba de tiempo contra la paz laboral. CENDES. UCV. Venezuela.

Irco, liese (2002). Universidad de Navarra y Accenture. Outsourcing del Recurso Humano. España.

Keil, thomas (2000). Strategic Alliances. A review of the state of the art. Working Paper Series 2000. No. 10. Helsinki University of Technology. Finland. Fuente: www.tuta.hut.fi. (Consultado el 16-04-2005).

Marín, Henry (2004). La gerencia del cambio en contextos de globalización. Revista de Ciencias Sociales. FACESLUZ. Vol. X. No. 1. pp. 9-27. Venezuela.

Monereo, José y Moreno, M. (2004). Las empresas de trabajo temporal en el marco de las nuevas formas de organización empresarial. Revista del Ministerio de trabajo y asuntos sociales. No. 48. pp. 39-65. España. Fuente: www.mtas.es (Consultado el 16-0606).

Mortimore, Michael y Pérez, W. (2001). La competitividad empresarial en América Latina y el Caribe. Revista de la CEPAL. No. 74. Fuente: www.eclac.cl (Consultado el 19-03-2007) 
Lucena, Héctor (2002). Un marco explicativo de la flexibilización laboral y de sus aplicaciones en Venezuela. Revista Principia. No. 19. UCLA. pp. 24-30. Venezuela.

Lucena, Héctor (2004). Relaciones de trabajo en el Nuevo Siglo. Fondo Editorial Tropykos. Venezuela.

Lucena, Héctor (2007). Lo laboral en tiempos de transición. Universidad de Carabobo. Valencia. Venezuela.

Ozaki, Muneto (2000). Negociar la flexibilidad. Función de los interlocutores sociales y el Estado. OIT. Ginebra.

Pin, J. y Suárez, E. (2004). La externalización de la Administración de Personal en la estrategia de Recursos Humanos. IRCO-IESE. Universidad de Navarra. España. Fuente: www.esadp.com (Consultado el 26-01-06).

Pirela, Arnoldo (2005). La apertura petrolera y el potencial de alianzas entre PDVSA y sus proveedores: El papel del contexto nacional. Revista Espacios. Vol. 26. Fuente: www.revistaespacios.com (Consultado el 14-09-2006).
Sáez, Francisco y Pineda, José (2004). Productividad y crecimiento en Venezuela: Un marco de referencia. BCV. Serie Documentos de Trabajo. $N^{\circ}$ 61. Fuente: www.bcv.org (Consultado el 19-03-2007)

Stuart, Toby (2000). Interorganizational alliances and the performance of firms: a study of growth and innovation rates in a high-technology industry. Strategic Management Journal. No. 21. p. 791-811.

Urrea, Fernando (1999). Un modelo de flexibilización laboral bajo el terror del mercado. Universidad de Cali. Colombia. En DE LA GARZA, ENRIQUE. 1999. Los retos teóricos de los estudios del trabajo hacia el siglo XXI. CLACSO. pp. 185-203. Argentina.

Wilson, David and Vlosky, R. (1998). Interorganizational information systems technology and buyer-seller relationships. The journal of Business \& Industrial Marketing. Tomo 13. No. 3. 